\title{
STUDY ON THE CONTENT OF GENIPOSIDIC ACID IN DIFFERENT PARTS OF EUCOMMIA ULMOIDES ON KARST PLATEAU OF GUIZHOU PROVINCE, CHINA
}

\author{
QIAN, C. J. ${ }^{1,2}-$ OU, M. C. ${ }^{2}-$ YU, L. F. ${ }^{1 *}-$ YAN, L. B. $^{1}-$ HUANG, Z. S. ${ }^{1}-$ FU, Y. H. ${ }^{2}$ \\ ${ }^{I}$ Guizhou University, The Key laboratory of Plant Resource Conservation and Germplasm \\ Innovation in Mountainous Region (Ministry of Education), Collaborative Innovation Center \\ for Mountain Ecology \& Agro-Bioengineering (CICMEAB) Guiyang 550025, Guizhou, China \\ ${ }^{2}$ Guizhou Education University, Guiyang 550018, Guizhou, China \\ *Corresponding author \\ e-mail: gdyulifei@163.com
}

(Received $15^{\text {th }}$ Feb 2020; accepted $7^{\text {th }}$ Jul 2020)

\begin{abstract}
This study aims to explore the content of (geniposidic acid, GPA) in the leaves, trunk bark and root bark of Eucommia ulmoides on Xingyi karst plateau mountains of Guizhou Province, China. There was no significant difference in the content of GPA in Eucommia ulmoides planted in different directions on the same slope. The content of GPA in leaves, trunk bark and root bark of Eucommia ulmoides planted on the sunny slope varied significantly, where the GPA content in root bark was the highest $(32.7851 \mathrm{mg} / \mathrm{g})$, There was no significant difference in GPA content in leaves and root bark of Eucommia ulmoides planted on the shady slope, but the GPA content in trunk bark was significantly different from that in leaves and root bark, where the GPA content in trunk bark was the highest $(30.3958 \mathrm{mg} / \mathrm{g})$, There was no significant difference in GPA content in trunk bark and root bark of Eucommia ulmoides planted in different slope directions, and the GPA content in leaves of Eucommia ulmoides planted on shady slope was significantly (36.71\%) higher than that on sunny slope. The GPA content in different parts of Eucommia ulmoides on both sunny or shade slope was GAP content in root bark was the largest followed by that in leaves, and that in trunk bark was the lowest. These results expand the source sites and provide new ideas for the comprehensive utilization and further research of Eucommia ulmoides resources.
\end{abstract}

Keywords: sunny slope and shady slope, different directions, root bark, trunk bark, leaves, high performance liquid chromatography

\section{Introduction}

The karst area in southwest China is the largest karst core area in the world (Wang et al., 2013). The exposed area of carbonate rocks in Guizhou is $12.8 \times 10^{4} \mathrm{~km}^{2}$, accounting for $73 \%$ of the total area of the province, which makes Guizhou the province with the most developed karst landform in China. Of the province's 86 counties (cities), 68 have more than 50\% karst area. Environmental degradation is prominent in karst areas (Cai, 1996), and problems such as soil-forming difficulties, lack of transition layer of limestone formed by carbonate rocks (Yang, 1990), thin soil, discontinuous soil cover and low ecological capacity also need to be addressed (Li et al., 2016). There are many factors restricting plant growth and development in karst environment. The central production areas of Eucommia ulmoides Oliver include Guizhou, Sichuan, Hubei, Hunan, Shaanxi and Henan (Li et al., 2001). The Eucommia ulmoides does not have strict requirements on environment and soil, and can grow on fertile hills, plains, barren red soil and harsh rock cliffs (Jiao, 2016). The Eucommia ulmoides is characterized by cold tolerance, drought resistance, poverty resistance, which can be 
grown in a wide range of area (Liu, 2012) with soil $\mathrm{pH}$ ranging from 5.0 to 8.4 (Du, 2003). There are plantations of Eucommia ulmoides in the harsh environment of Guizhou karst plateau.

As a unique deciduous tree of single species and single genus in China, The Eucommia ulmoides is a rare medicinal material unique to China as well asa widely used plant tonic. Eucommia ulmoides tea is a popular beverage in Asia and a new dualpurpose food resource for blood pressure medicine (Bai et al., 2015; Zhu and Sun, 2018). The Eucommia ulmoides contain abundant chemical constituents such as cycloene ether terpenes, bphenylpropanoids, flavonoids and phenols, which have a variety of medicinal values (Hussain et al., 2016; Yan et al., 2018). The Medicinal components of Eucommia ulmoides have anti-diabetes, anti-inflammatory, blood pressure lowering and diuretic effects (Peng and Li, 2013; Sugawa et al., 2016; Wang et al., 2016), anti-obesity effect (Hirata et al., 2011), anti-virus effect (Sun et al., 2004), effect of liver protection and gallbladder protection (Lou et al., 2011), and effect of improving hyperuricemia (Fang et al., 2019). The total flavonoids of Eucommia ulmoides have inhibitory effects on cell proliferation, migration and invasion of glioblastoma, one of the malignant primary brain tumors (Wang et al., 2019). The trunk bark extract of Eucommia ulmoides can reduce the serum level in liver injury (Lee et al., 2014). The leaf extract of Eucommia ulmoides has the effect of treating non-alcoholic fatty liver disease (Lee et al., 2019), and can be used as a new drug for treating male erectile dysfunction caused by diabetes (Fu et al., 2019). The GPA, chlorogenic acid, ginipinidine, rosin diglucoside, rutin and quercetin are the main components of Eucommia ulmoides (He et al., 2014). The GPA as the representative ingredient of terpenoids has the functions of anticancer, lowering blood pressure (Du et al., 2011). The GPA can change bile composition and prevent the formation of cholesterol stones (Huang et al., 2002), with anti-mutation activity and antiviral effect (Ong and Tan, 2007). Therefore, the studies on Eucommia ulmoides mainly focus on the pharmacology and the medicinal components of the bark and leaves of Eucommia ulmoides. In this study, the high-performance liquid chromatography (HPLC) was used to determine the content of GPA in leaves, trunk bark and root bark of Eucommia ulmoides in karst plateau mountains. The influence of different slope directions on the content of GPA in three parts as well as the content of GPA in leaves of Eucommia ulmoides planted in different slope directions were analyzed, with the purpose of providing a new idea for expanding the source site of Eucommia ulmoides.

\section{Materials and methods}

The Eucommia ulmoides samples were collected from Pishanlin village, Jingnan town, Xingyi city, Guizhou province, China (Fig. 1), which is a typical karst plateau mountainous area with a subtropical humid monsoon climate, where the soil type is lime soil, the average annual temperature is $16.1^{\circ} \mathrm{C}$ and the average annual rainfall is $1531.6 \mathrm{~mm}$. The longitude of the sunny slope is $104^{\circ} 49^{\prime} 11^{\prime \prime} \mathrm{E}$, the latitude is $24^{\circ} 55^{\prime} 29^{\prime \prime}$ $\mathrm{N}$, and the altitude is $1670 \mathrm{~m}$; The longitude of the shady slope is $104^{\circ} 52^{\prime} 21^{\prime \prime} \mathrm{E}$, the latitude is $24^{\circ} 55^{\prime} 20^{\prime \prime} \mathrm{N}$, and the altitude is $1610 \mathrm{~m}$.

The Eucommia ulmoides was planted in 1996 as a pure forest, and the samples collection date was May 29, 2017. Three $20 \mathrm{~m} \times 20 \mathrm{~m}$ quadrats were set on the sunny slope and the shady slope, respectively, with a total of 6 quadrats. According to the kraft grading principle, three dominant trees were selected from each quadrat, including a 
total of 18 plants of Eucommia ulmoides, of which the leaves, trunk bark and root bark were collected. The Eucommia ulmoides leaves without pests and diseases were selected from the east, south, west and north of the tree canopy. The trunk bark samples of Eucommia ulmoides were collected by ring stripping. The ring was cut $130 \mathrm{~cm}$ from the ground, and the second incision was cut $30 \mathrm{~cm}$ upward from this point. All the openings were cut longitudinally between the two incisions to obtain the trunk bark. The root bark samples were collected from the rough root. After digging out all the Eucommia ulmoides roots, the root bark was peeled off after removing the soil (Fig. 2). There were 36 samples of Eucommia ulmoides leaves on the sunny slope and the shady slope, respectively; there were 9 samples of Eucommia ulmoides trunk bark on the sunny slope and on the shady slope, respectively; there were 9 samples of Eucommia ulmoides root bark on the sunny slope and on the shady slope, respectively. In total, there were 108 samples. The samples of leaf, trunk bark and root bark of Eucommia ulmoides were placed in the self-sealing bags, labeled with the corresponding sample name, and then dried in the lab before use.

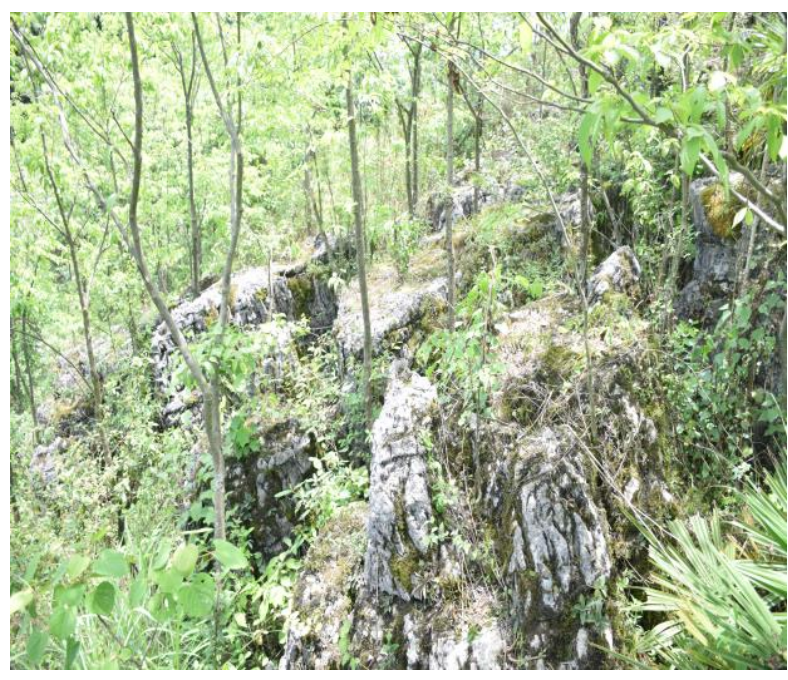

Figure 1. The habitat of Eucommia ulmoides

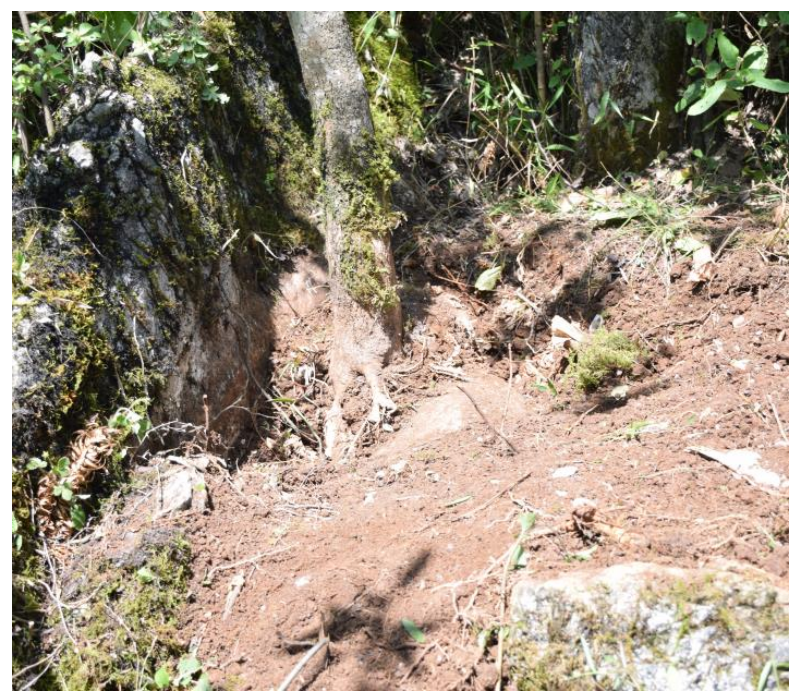

Figure 2. Digging the root of Eucommia ulmoides dominant wood 
The apparatuses used in the experiment include a single channel pipette (I53066G, R39656F; Eppendorf China Co., Ltd.), the circulating water type multipurpose vacuum pump (SHZ - D (III) (single; Shanghai to the China Instrument and Equipment Co., Ltd.), Agilent1260 high performance liquid chromatograph (Agilent Technology Co., Ltd.), chromatographic column (Agilent ZORBAX Eclipse Plus C18; Agilent Technology Co., Ltd.), electronic balance (Accuracy: Ten thousandth, ATY224; Shanghai Shengke Instrument Co., Ltd.), ultrasonic cleaner (SG8200HPT; Shanghai Guante Ultrasonic Instrument Co., Ltd.), electric blast drying oven (101-3A), highspeed universal pulverizer (FW80, Tianjin Taisite Instrument Co., Ltd.), low-speed multi-tube frame automatic balancing centrifuge (TDZ5-WS; Hunan Xiangyi Laboratory Instrument Development Co., Ltd.), ultra-pure water meter (Direct-Q8 UV system; Shanghai Merck Chemical Technology Co., Ltd.)

\section{Research methods and data analysis}

\section{Chromatographic condition}

The chromatographic column was ZORBAX Eclipse Plus C18 column $(4.6 \times 250 \mathrm{~mm}$, $5 \mu \mathrm{m}$; Agilent); The column temperature was $30{ }^{\circ} \mathrm{C}$. The mobile phase was acetonitrile (A) $-0.1 \%$ phosphoric acid solution (B) (97:3). Injection quantity $5 \mu \mathrm{L}$, Volume flow rate $1.0 \mathrm{~mL} / \mathrm{min}$; Detection wavelength $235 \mathrm{~nm}$ (Lv et al., 2012; Jiang et al., 2013; Qing et al., 2018). Qualitative test was conducted according to the retention time of the reference solution, and GPA content was calculated according to the peak area.

\section{Preparation of reference solution}

The GPA reference sample was weighed and dried to constant weight $(0.2200 \mathrm{mg})$ in a $120^{\circ} \mathrm{C}$ oven and placed in a $10 \mathrm{~mL}$ brown volumetric bottle. Then, the solution was dissolved with addition of methanol, diluted, and shaken well to obtain a GPA $0.0220 \mathrm{mg} / \mathrm{mL}$ control solution, which was stored in a refrigerator at $4{ }^{\circ} \mathrm{C}$ for later use (Lv et al., 2012; Jiang et al., 2013; Qing et al., 2018).

\section{Preparation of sample solution}

The samples of dried leaves, trunk bark and root bark of Eucommia ulmoides (the weight of each sample was $1.0000 \mathrm{~g}$ ) were placed in $250 \mathrm{~mL}$ conical flasks, and then $50 \mathrm{~mL}$ of $70 \%$ methanol were added for each flask. Ultrasonic treatment was performed for $30 \mathrm{~min}\left(30{ }^{\circ} \mathrm{C}, 300 \mathrm{~W}, 40 \mathrm{~Hz}\right)$ for extraction. The solution was transferred to a $100 \mathrm{~mL}$ centrifuge tube, centrifuged $(4000 \mathrm{r} / \mathrm{min})$ for $60 \mathrm{~min}$, filtered and diluted to $50 \mathrm{~mL}$, mixed well. After that, the solution was subjected to a $0.45 \mu \mathrm{m}$ Millipore filter before collecting filter liquor and transferring it to a $2.5 \mathrm{~mL}$ injection bottle to obtain the sample solution (Lv et al., 2012; Jiang et al., 2013; Qing et al., 2018).

\section{Data processing}

After the experimental data were input into EXCEL2010 for preliminary sorting, SPSS24.0 statistical software was used for data analysis. The One-Way ANOVA and LSD method were used for pair-wise comparison between groups (Tamhane's T2 was used if the variances were not equal). The difference was considered statistically significant when $\mathrm{p}<0.05$. 


\section{Results and analysis}

\section{Effect of slope direction on GPA content in different parts of Eucommia ulmoides}

The average GPA content in leaves, trunk bark and root bark of Eucommia ulmoides planted on sunny slope was $18.8755 \mathrm{mg} / \mathrm{g}, 9.8154 \mathrm{mg} / \mathrm{g}$ and $32.7851 \mathrm{mg} / \mathrm{g}$, respectively, where the average GPA content in root bark was the highest, which was 1.73 times that in leaves and 3.34 times that in trunk bark. The average GPA content in leaves, trunk bark and root bark of Eucommia ulmoides planted on shady slope was $29.8228 \mathrm{mg} / \mathrm{g}, 12.4351 \mathrm{mg} / \mathrm{g}, 30.3958 \mathrm{mg} / \mathrm{g}$, respectively, where the average GPA content in root bark was the highest, which was 1.02 times that in leaves and 2.44 times that in trunk bark. The average GPA content in leaves and trunk bark of Eucommia ulmoides planted on shady slope was $36.71 \%$ and $21.07 \%$ higher than that of Eucommia ulmoides planted on sunny slope. However, the average GPA content in root bark of Eucommia ulmoides planted on sunny slope was $7.29 \%$ higher than that planted on shady slope (Fig. 3).

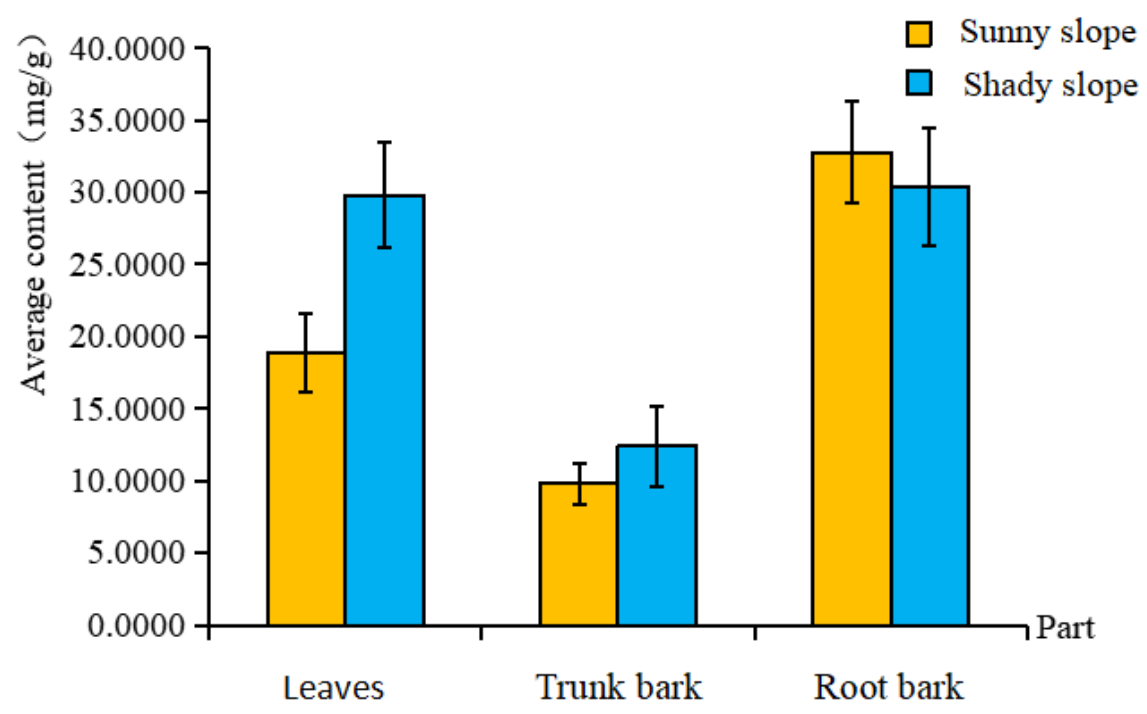

Figure 3. The Average GPA content in different parts of Eucommia ulmoides. The GPA content in different parts is an average value of 9 Eucommia ulmoides plants, GPA content in leaves is the average value of 36 samples in 4 directions of 9 plants; The error line in the figure is made by the standard deviation value obtained by SPSS software analysis, the following is the same

Of all Eucommia ulmoides plants planted on sunny slope of 3 quadrats, the east, south and west leaves of three Eucommia ulmoides plants planted on sunny slope of quadrat 2 had the largest average GPA contents, which was $32.8512 \mathrm{mg} / \mathrm{g}$ in the east, $24.8759 \mathrm{mg} / \mathrm{g}$ in the south and $22.7506 \mathrm{mg} / \mathrm{g}$ in the west, respectively (Table 1). Of all Eucommia ulmoides plants planted on shady slope of 3 quadrats, the east, north and west leaves of three Eucommia ulmoides plants planted on sunny slope of quadrat had the largest average GPA contents, which was $41.3838 \mathrm{mg} / \mathrm{g}$ in the east, $38.8765 \mathrm{mg} / \mathrm{g}$ in the north and $32.8861 \mathrm{mg} / \mathrm{g}$ in the west, respectively (Table 1). The GPA contents in the leaves in different directions of Eucommia ulmoides on the shady slope were generally higher than those on the sunny slope, 32.15\% higher in the east direction, 31.98\% higher in the south direction, $30.97 \%$ higher in the west direction, $51.27 \%$ higher in the north direction, and $36.71 \%$ higher on average (Fig. 4). 
Table 1. The GPA contents of Eucommia ulmoides leaves in different directions (unit: $\mathrm{mg} / \mathrm{g}$ )

\begin{tabular}{|c|c|c|c|c|c|c|c|c|}
\hline \multirow{2}{*}{ Sample name } & \multicolumn{2}{|r|}{ East } & \multicolumn{2}{|r|}{ South } & \multicolumn{2}{|r|}{ West } & \multicolumn{2}{|r|}{ North } \\
\hline & \begin{tabular}{|l|} 
Content \\
\end{tabular} & Average content & Content & Average content & Content & Average content & Content & Average content \\
\hline Sunny slope 1-1 & 15.6373 & \multirow{3}{*}{20.8990} & 22.1938 & \multirow{3}{*}{23.4366} & 15.0186 & \multirow{3}{*}{22.2403} & 18.2852 & \multirow{3}{*}{16.4362} \\
\hline Sunny slope $1-2$ & 26.1185 & & 22.8177 & & 20.8589 & & 12.4141 & \\
\hline Sunny slope 1-3 & 20.9413 & & 25.2982 & & 30.8435 & & 18.6092 & \\
\hline Sunny slope 2-1 & 44.9994 & \multirow{3}{*}{32.8512} & 34.4299 & \multirow{3}{*}{24.8759} & 28.4599 & \multirow{3}{*}{22.7506} & 26.8174 & \multirow{3}{*}{14.3284} \\
\hline Sunny slope $2-2$ & 32.0135 & & 24.3969 & & 22.5436 & & 12.4932 & \\
\hline Sunny slope 2-3 & 21.5406 & & 15.8008 & & 17.2484 & & 3.6747 & \\
\hline Sunny slope 3-1 & 10.4001 & \multirow{3}{*}{11.4774} & \begin{tabular}{|l|}
7.2360 \\
\end{tabular} & \multirow{3}{*}{11.6257} & 6.4477 & \multirow{3}{*}{11.9259} & 8.7534 & \multirow{3}{*}{13.6592} \\
\hline Sunny slope 3-2 & 19.7992 & & 19.5079 & & 18.5480 & & 23.1472 & \\
\hline Sunny slope 3-3 & 4.2329 & & 8.1332 & & 10.7820 & & 9.0769 & \\
\hline Shady slope 1-1 & 37.1771 & \multirow{3}{*}{41.3838} & 27.0310 & \multirow{3}{*}{30.9979} & 30.1355 & \multirow{3}{*}{32.8861} & 24.9982 & \multirow{3}{*}{38.8765} \\
\hline Shady slope 1-2 & 40.4021 & & 26.7918 & & 24.5900 & & 44.9588 & \\
\hline Shady slope 1-3 & 46.5721 & & 39.1708 & & 43.9328 & & 46.6726 & \\
\hline Shady slope 2-1 & 26.3005 & \multirow{3}{*}{24.8509} & 37.5194 & \multirow{3}{*}{25.5007} & 41.8741 & \multirow{3}{*}{24.5347} & 35.7397 & \multirow{3}{*}{28.2587} \\
\hline Shady slope 2-2 & 11.6734 & & 7.3637 & & 11.2789 & & 13.6083 & \\
\hline Shady slope 2-3 & 36.5787 & & 31.6190 & & 20.4510 & & 35.4281 & \\
\hline Shady slope 3-1 & 46.4620 & \multirow{3}{*}{29.8985} & 41.4126 & \multirow{3}{*}{31.6244} & 35.5867 & \multirow{3}{*}{25.0267} & 39.1191 & \multirow{3}{*}{24.0347} \\
\hline Shady slope 3-2 & 24.0767 & & 34.4809 & & 26.3403 & & 19.8311 & \\
\hline Shady slope 3-3 & 19.1569 & & 18.9797 & & 13.1531 & & 13.1538 & \\
\hline
\end{tabular}

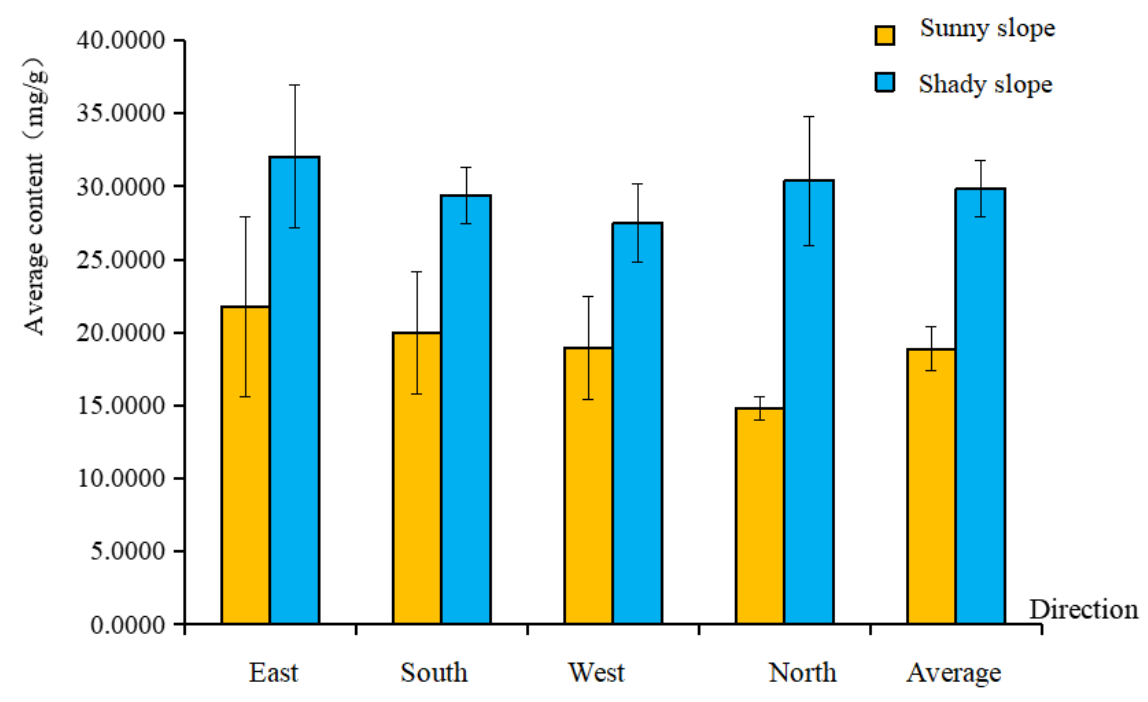

Figure 4. The average GPA contents of leaves in different directions of Eucommia ulmoides on sunny slope and shady slope. The GPA content of leaves in different directions of Eucommia ulmoides is the mean value of the content in corresponding directions of 9 plants (9 samples)

There was no significant difference in the GPA content of leaves of Eucommia ulmoides in different directions $(\mathrm{p}>0.05)$. The GPA content of leaves in different directions on the sunny slope was $21.7425 \mathrm{mg} / \mathrm{g}$ in the east, $19.9794 \mathrm{mg} / \mathrm{g}$ in the south, $18.97234 \mathrm{mg} / \mathrm{g}$ in the west, $14.8079 \mathrm{mg} / \mathrm{g}$ in the north, respectively (Fig. 5). There was no significant difference in GPA content of leaves in different directions for Eucommia ulmoides planted on shady slope $(\mathrm{p}>0.05)$. The GPA content of leaves in different directions on the shady slope was $32.0444 \mathrm{mg} / \mathrm{g}$ in the east, $29.3743 \mathrm{mg} / \mathrm{g}$ in the south, 
$27.4825 \mathrm{mg} / \mathrm{g}$ in the west, $30.3900 \mathrm{mg} / \mathrm{g}$ in the north, respectively, which is slightly different from the results of Eucommia ulmoides planted on sunny slope (Fig. 5).

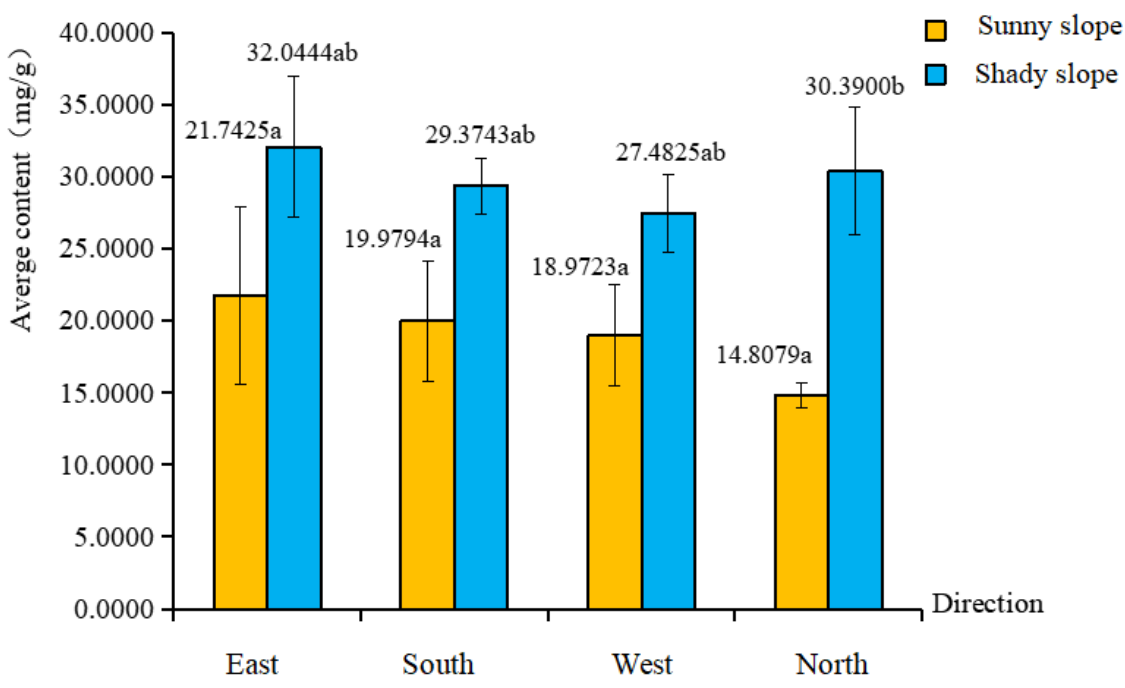

Figure 5. The difference analysis of GPA content in Eucommia ulmoides leaves in different directions on the sunny slope and the shady slope. The GPA content of Eucommia ulmoides leaves in each direction is the average value of 9 samples in the corresponding direction of 9 plants

There was a significant difference in GPA content in leaves, trunk bark and root bark of Eucommia ulmoides planted on sunny slope ( $\mathrm{p}<0.05$ ), and the GPA in root bark was $42.43 \%$ higher than that in leaves and $70.06 \%$ higher than that in trunk bark $(\mathrm{p}<0.05)$. The GPA content in leaves was significantly higher than that in trunk bark $(\mathrm{p}<0.05)$ by $48.00 \%$ for of Eucommia ulmoides planted on sunny slope. The GPA content in root bark was the largest followed by that in leaves, and that in trunk bark was the lowest (Fig. 6).

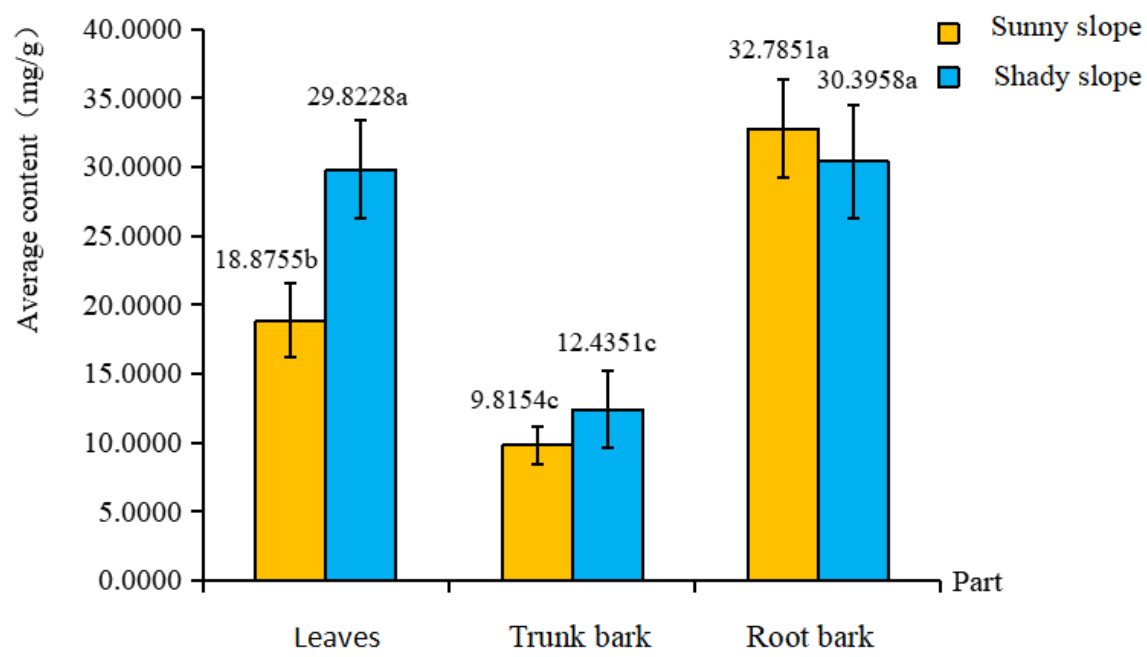

Figure 6. The difference analysis of GPA content in different parts of Eucommia ulmoides on the sunny slope and the shady slope. The GPA content in leaves was the mean value of 36 samples in 4 directions of 9 plants, while the GPA content in trunk bark and root bark content was the mean value of 9 plants 
The GPA contents in leaves and root bark of Eucommia ulmoides on shady slope showed no significant different $(\mathrm{p}>0.05)$. The GPA contents in leaves and root bark of Eucommia ulmoides on shady slope were significantly higher than GPA content in trunk bark by $58.30 \%$ and $59.09 \%$, respectively $(\mathrm{p}<0.05)$. The GPA content in different parts of Eucommia ulmoides on the shady slope in root bark was the largest followed by that in leaves, and that in trunk bark was the lowest (Fig. 6).

\section{Difference analysis of GPA contents in leaves of Eucommia ulmoides in different directions on sunny and shady slope}

There was no significant difference in GPA content of Eucommia ulmoides leaves in different directions on both sunny slope and the shady slope $(\mathrm{p}>0.05)$. In the east, south and west directions, there was no significant difference in GPA content in leaves of Eucommia ulmoides on both the sunny slope and the shady slope ( $p>0.05)$. In the north, the GPA content of Eucommia ulmoides leaves on the shady slope was $51.27 \%$ higher than that on the sunny slope $(p<0.05)$. In the different directions, the GPA content in Eucommia ulmoides leaves on the shady slope was higher than that on the sunny slope, which was $32.15 \%$ higher in the east, $31.98 \%$ higher in the south, $30.97 \%$ higher in the west, and $51.27 \%$ higher in the north (Fig. 5).

\section{Difference analysis of GPA content in different parts of Eucommia ulmoides on sunny slope and shady slope}

There was a significant difference in GPA content in leaves, trunk bark and root bark of Eucommia ulmoides on the sunny slope ( $<$ < 0.05). For Eucommia ulmoides on shady slope, there was no significant difference between the GPA content in leaves and that in root bark ( $p>0.05)$, while the GPA content in trunk bark was significantly different from that in leaves and that in root bark ( $<<0.05)$. The GPA content in Eucommia ulmoides leaves on shady slope was $36.71 \%$ higher than that on sunny slope $(\mathrm{p}<0.05)$. There was no significant difference between GPA content in trunk bark of Eucommia ulmoides on sunny slope and that on shady slope $(\mathrm{p}>0.05)$. There was no significant difference between the GPA content in root bark of Eucommia ulmoides on sunny slope and that on shady slope $(p>0.05)$. There was a significant difference in GPA content in leaves between Eucommia ulmoides on shady slope and that on sunny slope, the GPA content in leaves and trunk bark of Eucommia ulmoides on shady slope was higher than that on sunny slope by $36.71 \%$ and $21.07 \%$, respectively. The GAP content in root bark of Eucommia ulmoides on sunny slope was $7.29 \%$ higher than that on shady slope (Fig. 6).

\section{Discussion}

At present, the Eucommia ulmoides-related researches are mainly focused on the GPA contents in leaves and bark (Xu, 2007; Wei, 2016; Yan, 2018), the GPA content in seeds (Liu, 2013) and the GPA content in male flowers (He, 2010). However, there have been no systematic study on the GPA contents in leaves, trunk bark and root bark of Eucommia ulmoides in different directions on the sunny slope and the shady slope. In the karst plateau mountainous area studied, the GPA content in different parts of Eucommia ulmoides on both sunny or shade slope was GAP content in root bark was the largest followed by that in leaves, and that in trunk bark was the lowest. This is not consistent with the study (Jiang et al., 2013) which reports that the GPA content in the 
bark of Eucommia ulmoides from the same origin is higher than that in the leaves of Eucommia ulmoides, and also not consistent with the study (Lv et al., 2012) which reports that the GPA content of GPA in the bark of Eucommia ulmoides is higher than that in the leaves in Hunan province. Such different may be related to differences in sampling time and habitats of Eucommia ulmoides in different areas. In this study, the GPA content of Eucommia ulmoides root bark was studied for the first time, and it was found that the GPA content of Eucommia ulmoides root bark is higher than that of trunk bark and leaves, which may have higher medicinal value of root bark. Currently, the root bark of Eucommia ulmoides has not been included in the 2015 edition of Chinese pharmacopoeia as the medicinal part. Further study on the GPA content of root bark of Eucommia ulmoides will have important guiding significance for making full use of Eucommia ulmoides resources.

\section{Conclusion}

In the karst plateau mountains where Eucommia ulmoides samples are collected, the GPA content in Eucommia ulmoides leaves in different directions on shady slope was generally $36.71 \%$ higher than that on sunny slope. There was no significant difference in the GPA content in leaves in different directions for Eucommia ulmoides on the same slope direction. Except that the GPA of Eucommia ulmoides leaves in the north on the shady slope was significantly higher (by 51.27\%) than that in the north of the sunny slope, there was no significant difference in the GPA of Eucommia ulmoides leaves in the other three directions. Therefore, if GPA in the Eucommia ulmoides leaves is the target medicinal component, it is most suitable to collect Eucommia ulmoides leaves in the north on shady slope.

The GPA contents in different parts of Eucommia ulmoides on sunny are the GPA content in root bark $(32.7851 \mathrm{mg} / \mathrm{g})$, that in leaves $(18.8755 \mathrm{mg} / \mathrm{g})$ and that in trunk bark $(9.8154 \mathrm{mg} / \mathrm{g})$. In contrast, the results are GPA content in the root bark $(30.3958 \mathrm{mg} / \mathrm{g})$, that in the leaf $(29.8228 \mathrm{mg} / \mathrm{g})$, that in the trunk bark $(12.4351 \mathrm{mg} / \mathrm{g})$ for Eucommia ulmoides on shady slope.

The GPA content in different parts of Eucommia ulmoides on both sunny or shade slope was GAP content in root bark was the largest followed by that in leaves, and that in trunk bark was the lowest. If the GPA in Eucommia ulmoides is taken as the target component, the root bark is the best resource. This study expands the medicinal source part of Eucommia ulmoides and provides a new idea for the comprehensive utilization of Eucommia ulmoides resources. In the later stage, it will strengthen the research on the content of other medicinal components in the root bark, trunk bark and leaves of Eucomia ulmoides on the karst plateau mountain areas, further explore the influencing factors of the content of medicinal components in different parts of Eucomia ulmoides in the harsh karst environment, and explore the relationship between the biomass and the content of medicinal components in each part.

Acknowledgements. This work is supported by the 13th Five-year National Key Research and Development Plan (2016YFC0502604), Construction Program of Biology First-class Discipline in Guizhou (GNYL[2017]009), the Project of Promoted Innovation of Colleges and Universities of Guizhou Province (Qian Jiao He Collaborative Innovation [2014]01), National Natural Science Foundation of China (51868008), Guizhou provincial first-class major (biological science) project (Education 
department of guizhou province[2019]46); and Guizhou First-class Teaching Team Construction Project (Qian Jiao Gao Fa [2017] 158)].

\section{REFERENCES}

[1] Bai, M. M., Shi, W., Tian, J. M., Zhang, K. J., Kim, J. H., Sun, Y. N., Kim, Y. H., Gao, J. M. (2015): Soluble epoxide hydro-lase inhibitory and anti-inflammatory components from the leaves of Eucommia ulmoides Oliver (duzhong). - Journal of Agricultural and Food Chemistry 63(8): 2198-2205.

[2] Cai, Y. L. (1996): Preliminary research ecological reconstruction in karst mountains poverty areas of Southwest China. - Advances in Earth Science 11(6): 602-606.

[3] Du, H. Y. (2003): The progress in research of the active component from Eucommia ulmoides and its pharmacology. - Economic Forest Researches 2: 58-61, 82.

[4] Du, H. Y., Li, Q., He, J. J., Liu, C. Y., Liu, P. F. (2011): Comparison of the main active components contents in barks of different variance types of Eucommia ulmoides. - Forest Research 24(02): 230-233.

[5] Fang, C., Chen, L. Y., He, M. Z., Luo, Y. Y., Zhou, M. J., Zhang, N., Yuan, J. F., Wang, H. L., Xie, Y. Y. (2019): Molecular mechanistic insight into the anti-hyperuricemic effect of Eucommia ulmoides in mice and rats. - Pharmaceutical Biology (57)1: 112-119.

[6] Fu, H., Bai, X., Le, L., Tian, D., Gao, H., Qi, L. X., Hu, K. D. (2019): Eucommia ulmoides Oliv. leaf extract improves erectile dysfunction in streptozotocin-induced diabetic rats by protecting endothelial function and ameliorating hypothalamic-pituitarygonadal axis function. - Evidence-Based Complementary and Alternative Medicine. https://doi.org/10.1155/2019/1782953.

[7] He, J. J. (2010): Studies on Variation of Secondary Metabolites in Eucommia Bark and Eucommia Male Flower. - Henan University, Henan.

[8] He, X., Wang, J. H., Li, M. X., Hao, D. J., Yang, Y., Zhang, C. L., He, R., Tao, R. (2014): Eucommia ulmoides Oliv.: ethnopharmacology, phytochemistry and pharmacology of an important traditional Chinese medicine. - Journal of Ethnopharmacology 151(1): 78-92.

[9] Hirata, T., Kobayashi, T., Wada, A., Ueda, T., Fujikawa, T., Miyashita, H., Ikeda, T., Tsukamoto, S., Nohara, T. (2011): Antiobesity compounds in green leaves of Eucommia ulmoides. - Bioorganic \& Medicinal Chemistry Letters 21(6): 1786-1791.

[10] Huang, R. H., Xiang, Y., Liu, X. Z., Zhang, Y., Hu, Z., Wang, D. C. (2002): Two novel antifungal peptides distinct with a five-disulfide motif from the bark of Eucommia ulmoides Oliver. - Federation of European Biochemical Societies 521(01): 87-90.

[11] Hussain, T., Tan, B. E., Liu, G., Oladele, O. A., Rahu, N., Tossou, M. C., Yin, Y. L. (2016): Health-promoting properties of Eucommia ulmoides: a review. - Evidence-Based Complementary and Alternative Medicine. https://doi.org/10.1155/2016/5202908.

[12] Jiang, X. F., Zhang, C. L., Li, Q., Du, H. Y., Du, L. Y., Fu, J. M. (2013): Determination of three active components in leaves and bark of Eucommia ulmoides from different habitats by RP-HPLC. - Jiangsu Agricultural Sciences 41(08): 314-316.

[13] Jiao, H. L. (2016): Study on the Extraction and Quality of Eucommia Ulmoides Seed Oil. - Henan University of Technology, Henan.

[14] Lee, G. H., Lee, M. R., Lee, H. Y., Kim, S. H., Kim, H. K., Kim, H. R., Chae, H. J. (2014): Eucommia ulmoides cortex, geniposide and aucubin regulate lipotoxicity through the inhibition of lysosomal BAX. - PLoS ONE 9(2): 1-14.

[15] Lee, G. H., Lee, H. Y., Park, S. A., Shin, T. S., Chae, H. J. (2019): Eucommia ulmoides leaf extract ameliorates steatosis induced by high-fat diet in rats by increasing lysosomal function. - Nutrient 11(426): 1-15.

[16] Li, F. D., Du, H. Y. (2001): Eucommia ulmoides Oliver. - China Press of Traditional Chinese Medicine, Beijing, pp. 260-280. 
[17] Li, J. X., Xiong, G. M., Xu, W. T., Zong, Q. X. (2016): Distribution of shrublands in relation to soil and climate in mid-subtropical China. - Journal of Plant Ecology 9(4): 393-401.

[18] Liu, P. F. (2012): Isolation, Identification and Sequence Characterization of Full Length cDNA of MEP Pathway Related Genes in Eucommia ulmoides. - Chinese Academy of Forestry, Beijing.

[19] Liu, T. T. (2013): The New Technology for Multistage Efficient Utilization of Eucommia Ulmoide Oliver. Resources. - Northeast Forestry University, Harbin.

[20] Lou, L. J., Chen, B. Q., Du, H. Y., Fu, J. M., Du, L. Y., Li, Q. (2011): Protection effects of Eucommia ulmoides male flower tea on carbon tetrachloride induced liver injury in mice. - Journal of Henan University (Medical Science) 30(1): 24-26.

[21] Lv, Q., Peng, M. J., Lan, W. J., Peng, S., Zhang, L. J. (2012): Effect of processing methods on the content of several active ingredients in barks and leaves of Eucommia ulmoides Oliver. - Chemistry and Industry of Forest Products32(01): 75-79.

[22] Ong, V. Y. C., Tan, B. K. H. (2007): Novel phytoandrogens and lipidic augmenters from Eucommia ulmoides. - BMC Complementary and Alternative Medicine 7(01): 1-11.

[23] Peng, H. M., Li, X. Z. (2013): Pharmacological research and application of duzhong. Acta Chinese Medicine 28(176): 72-73.

[24] Qing, J., Wei, Y. X., Wang, D. H., Wang, L., Liu, P. F., Du, H. Y., Du, Q. X. (2018): Study on genetic variation of main active components in leaves of Eucommia ulmoides. Acta Botanica Boreali-Occidentalia Sinica 38(2): 316-323.

[25] Sugawa, H., Ohno, R. I., Shirakawa, J. I., Nakajima, A., Kanagawa, A., Hirata, T., Ikeda, T., Moroishi, N., Nagaia, M., Nagai, R. (2016): Eucommia ulmoides extracts prevent the formation of advanced glycation end products. - Food \& Function 7(6): 2566-2573.

[26] Sun, Y. R., Dong, J. X., Wu, S. G. (2004): Studies on chemical constituents from Eucommia ulmoides Oliver. - Journal of Chinese Medicinal Materials 27(5): 341-343.

[27] Wang, J. W., Zhou, Y., Xiao, B. X., Li, J. S. (2013): Progress of study on karst soil moisture characteristics of Southwest China. - Soil and Water Conservation in China 2: $37-42$.

[28] Wang, J. Y., Yuan, Y., Chen, X. J., Fu, S. G., Zhang, L., Hong, Y. L., You, S. F., Yang, Y. Q. (2016): Extract from Eucommia ulmoides Oliv. ameliorates arthritis via regulation of inflammation, synoviocyte proliferation and osteoclastogenesis in vitro and in vivo. Journal of Ethnopharmacology 194: 609-616.

[29] Wang, Y. S., Tan, X. R., Li, S., Yang, S. L. (2019): The total flavonoid of Eucommia ulmoides sensitizes human glioblastoma cells to radiotherapy via HIF- $\alpha / \mathrm{MMP}-2$ pathway and activates intrinsic apoptosis pathway. - Onco Targets and Therapy (12): 5515-5524.

[30] Wei, Y. X. (2016): Study on variability of main active components and rubber content in Eucommia ulmoides. - Chinese Academy of Forestry 1-87.

[31] Xu, Y. M. (2007): The Effects of External Hormones on the Content of Secondary Metabolites in Eucommia ulmoides Oliver. - Northwest Agricultural and Forestry University of Science and Technology, Shaanxi.

[32] Yan, Y. (2018): Studies on quality evaluation of Eucommiae Cortex. - Nanjing University of Chinese Medicine 1-148.

[33] Yan, Y., Zhao, H., Chen, C. H., Zou, L. S., Liu, X. H., Chai, C., Wang, C. C., Shi, J. J., Chen, S. Y. (2018): Comparison of multiple bioactive constituents in different parts of Eucommia ulmoides based on UFLC-QTRAP-MS/MS combined with PCA. - Molecules 23(643): 1-11.

[34] Yang, M. D. (1990): On the fragility of the karst environment. - Yunnan Geographic Environment Research 2(01): 21-29.

[35] Zhu, M. and Sun, R. (2018): Eucommia ulmoides Oliver: a potential feedstock for bioactive products. - Journal of Agricultural and Food Chemistry 66(22): 5433-5438. 